\title{
Design Patterns for Helping Students to Learn to Represent Math Problems in Online Learning Systems
}

PAUL SALVADOR INVENTADO \& PETER SCUPELLI School of Design, Carnegie Mellon University

Online learning systems have been gaining popularity, but are not without their challenges. For example, enrollment in MOOCs has slowed down, which is attributed to the lack of sustainability. The success of online learning systems is heavily influenced by how they were designed. For example, results from a recent study showed that the incorporation of learning activities to instruction increased learning gains as much as six times. Although there are many design patterns that may be applied in the design of learning activities, they usually operate at a higher level. There is a need for design patterns that address problems in implementing learning activities in online learning systems. Specifically, the goal of the four design patterns presented is to help students learn to represent math problems. The patterns presented in this paper are part of a pattern language for creating math problems and corresponding learning support in online learning systems.

- Applied computing Distance learning • Human-centered computing User centered design

Additional Key Words and Phrases: Design patterns, online learning systems, problem solving, feedback, data driven design pattern production, ASSISTments

ACM Reference Format:

Paul Salvador Inventado and Peter Scupelli. 2016. Design Patterns for Helping Students to Learn to Represent Math Problems in Online Learning Systems. EuroPLoP'16. (July 06-10 2016), 19 pages.

DOI: http://dx.doi.org/10.1145/3011784.3011816

\section{INTRODUCTION}

Increasingly, online learning systems have been gaining popularity. Examples of such systems include Massively Open Online Courses (MOOCs) (e.g., Coursera, edX) and online tutoring systems (e.g., ASSISTments, Cognitive Tutor). Although MOOCs are still popular, enrollment in such courses have started to slow down because of their lack of sustainability [Allen and Seaman 2015].

Some online learning systems are more successful than others because of the designs that were used in their development. In a recent article by Koedinger and colleagues [2015], for example, they showed that incorporating learning activities (e.g., problem solving exercises) with instruction (e.g., view video lectures, read content) in MOOCs resulted in as much as six times more learning gains.

Uncovering design patterns from successful online learning systems can benefit various online learning system stakeholders (e.g., system developers, content creators, teachers) by guiding them in the creation of high quality systems. There are design patterns that can be used to design online learning system components such as flipped classroom patterns [Köppe et al. 2015], massive open online course (MOOC) patterns [Mor and Warburton 2015], and pedagogical patterns [Bergin et al. 2012], but they often operate at higher levels (e.g., classroom management, handling lectures, addressing technological issues). There are fewer design patterns that address specific aspects of learning in online learning systems (e.g., problem content, feedback content, problem sequence).

Permission to make digital or hard copies of all or part of this work for personal or classroom use is granted without fee provided that copies are not made or distributed for profit or commercial advantage and that copies bear this notice and the full citation on the first page. Copyrights for components of this work owned by others than the author(s) must be honored. Abstracting with credit is permitted. To copy otherwise, or republish, to post on servers or to redistribute to lists, requires prior specific permission and/or a fee. Request permissions from Permissions@acm.org.

EuroPLoP '16, July 06 - 10, 2016, Kaufbeuren, Germany

Copyright is held by the owner/author(s). Publication rights licensed to ACM.

ACM 978-1-4503-4074-8/16/07...\$15.00

DOI:http://dx.doi.org/10.1145/3011784.3011816 
The patterns presented in this paper are part of a pattern language for creating math problems and corresponding learning support in online learning systems, which is discussed in more detail in Section 4. More specifically, the patterns address problems related to students' difficulty with the representation of math problems and corresponding solutions (e.g., mathematical notations, equations, drawn figures), which is an essential skill in mathematics [Polya 2014]. Math teachers and content designers might find the patterns useful to guide the creation of high quality math learning activities in the context of an online learning system. Math is used in many other domains, so the presented patterns may also be useful for teachers and content designers working in related domains.

The following sections provide a review of related work, a brief discussion about the methodology used to develop the patterns presented in the paper, the design patterns, and future work.

\section{RELATED WORK}

There are several design patterns for the educational domain. Many of them comprise pattern languages that focus on particular educational contexts. For example, Köppe and colleagues [2015] are compiling design patterns for a flipped classroom pattern language. These patterns help with the design and delivery of in-class meetings to ensure that students get necessary support in deepening their understanding of the knowledge, and to correct possible misconceptions they acquired.

An increasingly popular educational context is online learning. Mor and Warburton are compiling a pattern language for MOOCs to address various challenges such as those involving course management, online discussions, interaction between learners, course content, individual differences, and failure points in the technology [Mor and Warburton 2015, Warbuton and Mor 2015].

Mor [2010] also compiled techno-pedagogical design patterns for teaching and learning mathematics through games, guided learning, and collaborative learning. These activities are facilitated by technological tools like exploratory learning environments, editable documents, and discussion boards.

Bergin and colleagues' [2012] pedagogical patterns are quite robust and can be applied in various educational contexts such as traditional learning settings, flipped classrooms, and MOOCs. The patterns address various problems on active learning, feedback, and experiential learning to name a few. Pedagogical patterns for experiential learning and feedback such as Try It Yourself, One Concept - Several Implementations, and Feedback, are particularly interesting because they can be applied in designing content and feedback for online learning activities [Bergin et al. 2012].

\section{DATA-DRIVEN DESIGN PATTERN DEVELOPMENT}

The design patterns presented in the following section were uncovered using the data-driven design pattern production (3D2P) methodology. 3D2P is a four-step iterative process used to uncover design patterns from data collected in a particular domain [Inventado and Scupelli 2015a]. As illustrated in Figure 1, 3D2P starts by prospecting data to find interesting relationships in the data. These relationships are investigated further in the pattern-mining step to develop hypotheses based on recurring problems and high quality solutions uncovered. Literature and experts in the field are consulted to test the validity of the hypotheses. Resulting hypotheses are used to write proposed patterns, which are further refined with the help of the design pattern community through mentoring and pattern writing workshops. Accepted design patterns are evaluated by implementing them into existing systems and evaluating their performance. Randomized controlled trials are conducted to compare the resulting outcome measures (e.g., learning gain, time on task) between applying the design pattern and not applying the design pattern. Results of the evaluation are used to further refine the design pattern as needed. Details about the methodology can be found in [Inventado and Scupelli, 2015a]. 


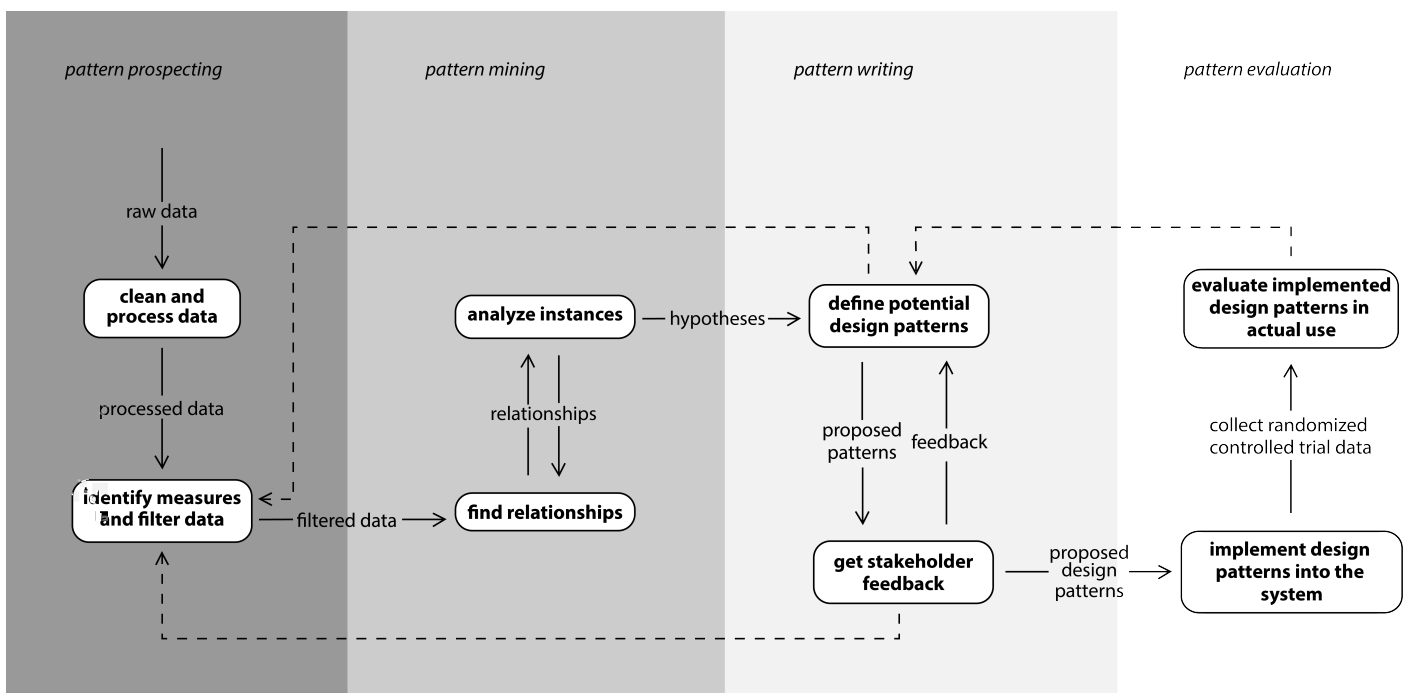

Figure 1. Data-driven design pattern production methodology (Image courtesy of the Learning Environments Lab).

\section{DESIGN PATTERNS}

Figure 2 illustrates the pattern language for creating math problems and corresponding learning support in online learning systems. The four patterns presented in this paper belong to this pattern language and can be used in conjunction with other patterns in the language.

The innermost rectangles in the figure represent design patterns. Published patterns are drawn using solid lines and patterns-in-development are drawn with dotted lines. Empty rectangles indicate that other patterns may later be included in the language. The design patterns are grouped into five categories represented by outer rectangles that enclose the patterns: Problems, Learning Support, Personalized Learning, Motivation, and Mastery Learning. Problems refer to patterns that deal with the design of math problem content. Learning Support refers to patterns that deal with the design of math learning-support content, how they are presented, or how much learning support should be provided. It is further categorized according to the type of learning support provided such as Hints, Examples, and Scaffolding. Personalized Learning refers to patterns that deal with designs for adapting problem content or learning-support content so that it is more appropriate to the student's background knowledge, affective state, personality, and so forth. Motivation refers to patterns that deal with designs for maintaining student motivation while maximizing learning. Finally, Mastery Learning refers to patterns that deal with the design of math problems or problem sets to promote skill mastery. Some of these design patterns have recently been published, which can be found in Inventado and Scupelli [2015a], Inventado and Scupelli [2015b], Inventado and Scupelli [2016a], and Inventado and Scupelli [2016b]. 

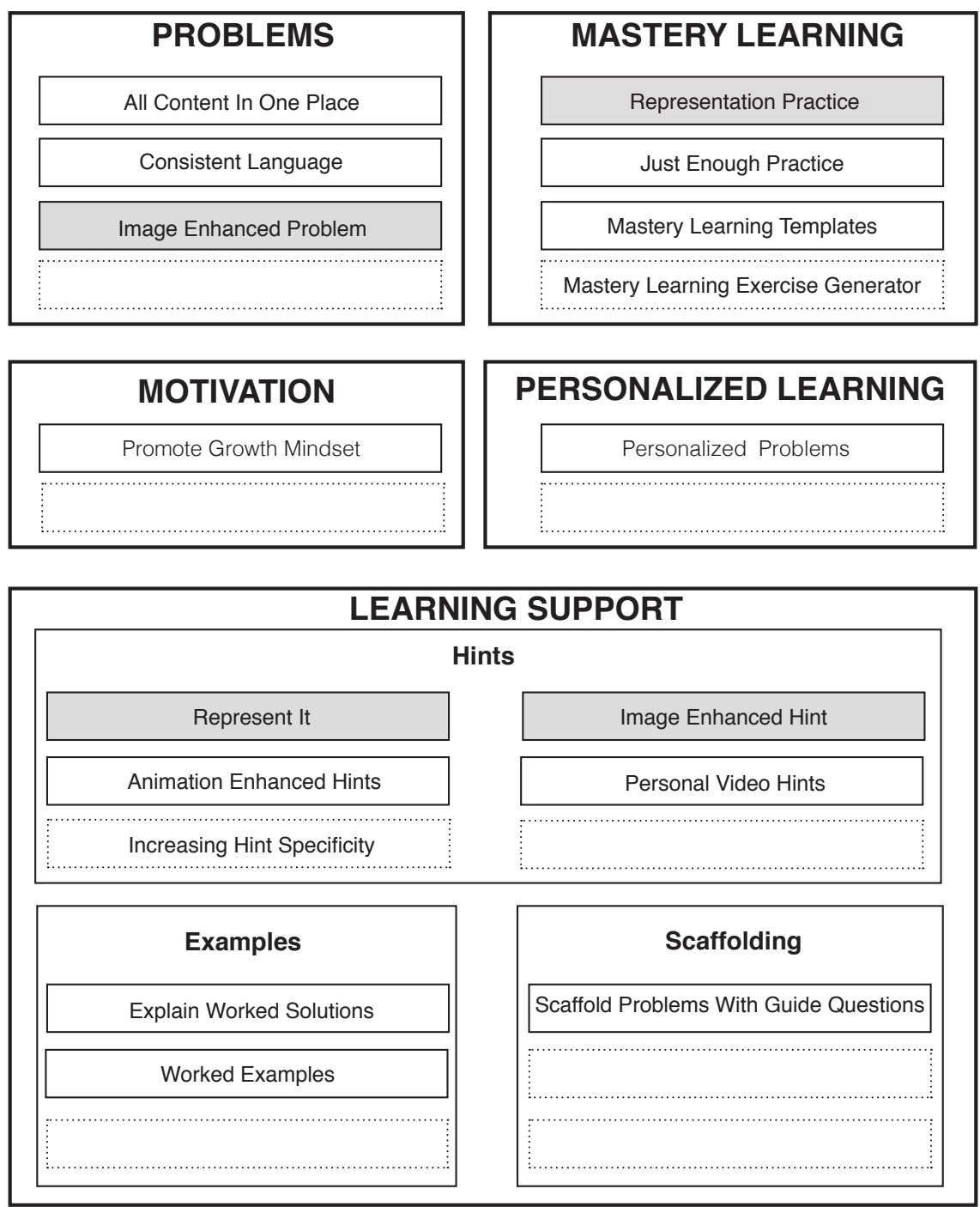

Fig. 2. Visualization of the Pattern Language for Math problems and Learning Support in Online Learning Systems (Image courtesy of the Learning Environments Lab).

The pattern format used in this paper separates each part with a heading much like other pattern formats (c.f., [Carlsson 2004, Dearden and Finaly 2006]). It contains the usual context, problem, forces, and solution sections. The benefits section describes how the solution addresses the forces in the problem and the liabilities section presents the issues that may arise from implementing the solution. The known implementations section describes instances when the pattern was applied successfully, and the related patterns section lists other design patterns that are referenced or references the pattern in question. A supporting theories section was added to allow the reader to learn more about the theoretical foundation of the problem and solution described in the pattern.

Table 1 summarizes the four patterns presented in the following subsections, while Table 2 summarizes referenced patterns. 
Table 1. Patterns for helping students represent math problems in online learning systems.

\begin{tabular}{|l|l|}
\hline Design Pattern & Summary \\
\hline Representation Practice & $\begin{array}{l}\text { Ask students to answer practice problems that require them } \\
\text { to use different representations so they gain experience and } \\
\text { hone their skills in selecting, applying, and utilizing } \\
\text { appropriate representations. }\end{array}$ \\
\hline Represent It & $\begin{array}{l}\text { Encourage students to externalize their thoughts using } \\
\text { representations to help them better understand the problem } \\
\text { and figure out the answer. }\end{array}$ \\
\hline Image-Enhanced Problem & $\begin{array}{l}\text { Clarify ambiguous terms and explanations in the math } \\
\text { problem by adding appropriate images. }\end{array}$ \\
\hline Image-Enhanced Hint & $\begin{array}{l}\text { Clarify hints for math problems by adding images that help } \\
\text { to disambiguate confusing terms and explanations. }\end{array}$ \\
\hline
\end{tabular}

Table 2. Referenced Patterns

\begin{tabular}{|l|l|}
\hline Referenced Design Patterns \\
\hline $\begin{array}{l}\text { Build and Maintain Confidence } \\
\text { [Bergin et al. 2012] }\end{array}$ & $\begin{array}{l}\text { Gummary } \\
\text { feedback, so they apply what they learned, discover there are } \\
\text { multiple solutions, and realize they can implement solutions } \\
\text { themselves. }\end{array}$ \\
\hline $\begin{array}{l}\text { Digestible Packets } \\
\text { [Bergin et al. 2012] }\end{array}$ & Organize topics so they are small and understandable. \\
\hline $\begin{array}{l}\text { Increasing Hint Specificity } \\
\text { (under development) }\end{array}$ & $\begin{array}{l}\text { Allow students to request for a series of hints, which increase } \\
\text { in specificity as more hints are requested. }\end{array}$ \\
\hline $\begin{array}{l}\text { Keep It Simple } \\
\text { [Cunningham and Cunningham 2014] }\end{array}$ & $\begin{array}{l}\text { Designs should be kept as simple as possible while ensuring } \\
\text { it still achieves its purpose. }\end{array}$ \\
\hline $\begin{array}{l}\text { One Concept - Several } \\
\text { Implementations } \\
\text { [Bergin et al. 2012] }\end{array}$ & $\begin{array}{l}\text { Present and compare several implementations of an abstract } \\
\text { concept so learners understand its essence and not just } \\
\text { associate it with a single implementation. }\end{array}$ \\
\hline $\begin{array}{l}\text { Try It Yourself } \\
\text { [Bergin et al. 2012] }\end{array}$ & $\begin{array}{l}\text { Ask students to answer exercises that will give them a better } \\
\text { understanding of the topic. }\end{array}$ \\
\hline
\end{tabular}




\section{Representation Practice}

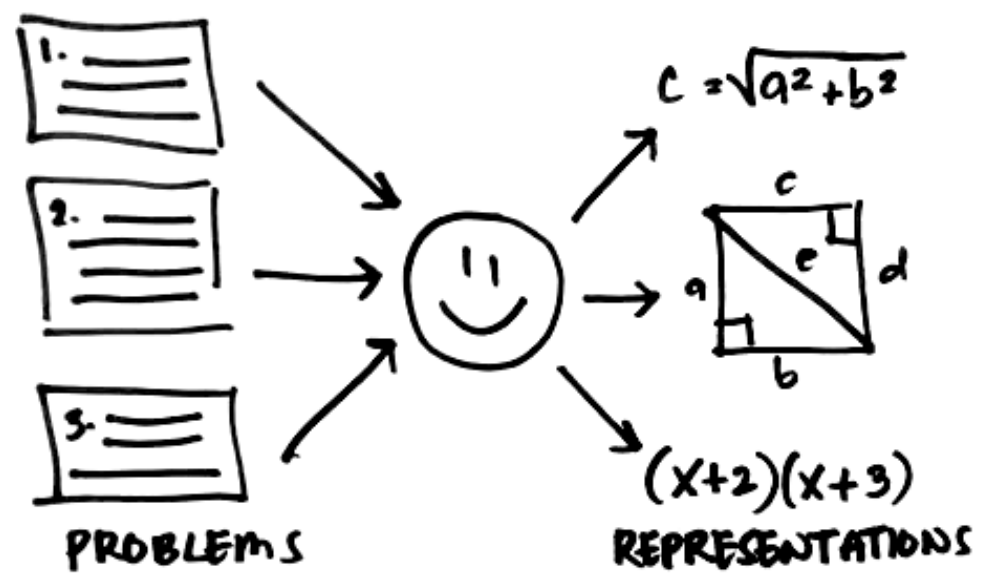

Context: Students recently started to learn about a new topic discussed in class.

Problem: Students get stuck when they don't know how to represent a problem or solution.

\section{Forces:}

- Lack of prior knowledge. Students cannot apply a representation that they have not seen or used.

- Availability bias. Students are likely to use representations that they can easily recall, but these may not be appropriate to the problem they are currently solving.

- Appropriate representation. Students may know about different types of representations, but they may not know how to select what is most appropriate to a given problem.

Solution: Therefore, use an online learning system to provide students with practice problems that require them to create and use different representations. For example, one practice problem's text may describe geometric shapes that would require students to draw the figure. Another problem may require students to recall and use an algebraic equation. Yet another problem may require students to do both.

Research shows that students learn better with frequent and spaced practice compared to massed practice. For example, give students practice problems that involve drawing isosceles triangles twice a week and mix it with problems that involve other representation types.

Students who have just started to learn using a particular representation might have a difficult time to answer practice problems. Providing students with worked examples and interleaving them with practice problems have been found to be effective. As students gain expertise worked examples can be faded so that they answer practice problems themselves.

The Represent It design pattern can help to encourage students use representations.

\section{Benefits:}

- Practice problems give students opportunities to learn about many different representations.

- Practice helps to build memory and fluency, which facilitates the recall of different representations. 
- As students practice on different representations, they begin to learn when, why, and how to apply them.

\section{Liabilities:}

- Teachers need to design and implement various practice problems on the online learning system for students to learn from.

- Students who continuously fail to answer problems may lose motivation. They may be more likely to give up when asked to solve similar problems. Worked examples or feedback may be needed to support learning.

- It takes time for students to learn and master skills for representing problems and solutions.

\section{Supporting Theories:}

- Practice promotes deeper understanding of skills that integrate with prior knowledge to develop expertise [Clark and Mayer 2011]. Experts from different domains devote significantly more time to practice compared to average and novice performers.

- Practice requires students to recall and apply information, which leads to better learning compared to instruction alone [Clark and Mayer 2011]. Frequent, but spaced practice leads to better learning because it allows students to forget information, but requires them to recall information in the next practice session [Clark and Mayer 2011, Rohrer and Taylor 2006].

- Students who are just starting to learn a particular topic may lack prior knowledge to answer practice problems. An effective strategy is to interleave worked examples and practice problems so that students learn how to perform a skill and get practice applying it themselves then gradually decrease examples and increase practice to develop students' skills [Renkl 2014].

Known implementations: Math teachers use notations, equations, and figures to clarify concepts they teach and encourage students to apply the same strategy when they solve practice exercises (e.g., [McIntosh 2015, Noh et al. 2013, Polya 2014]). Math online learning systems such as ASSISTments [Heffernan and Heffernan 2014], Cognitive Tutor Geometry [Aleven et al. 2006], and Picture Algebra [Koedinger 2002] also provide students with practice problems that contain many problem details, which students need to represent correctly to solve.

The following figure shows an example of a practice problem for "Understanding the Coordinate System" in the ASSISTments online learning system. It may be difficult to solve the problem mentally, so it encourages the student to draw a figure. 


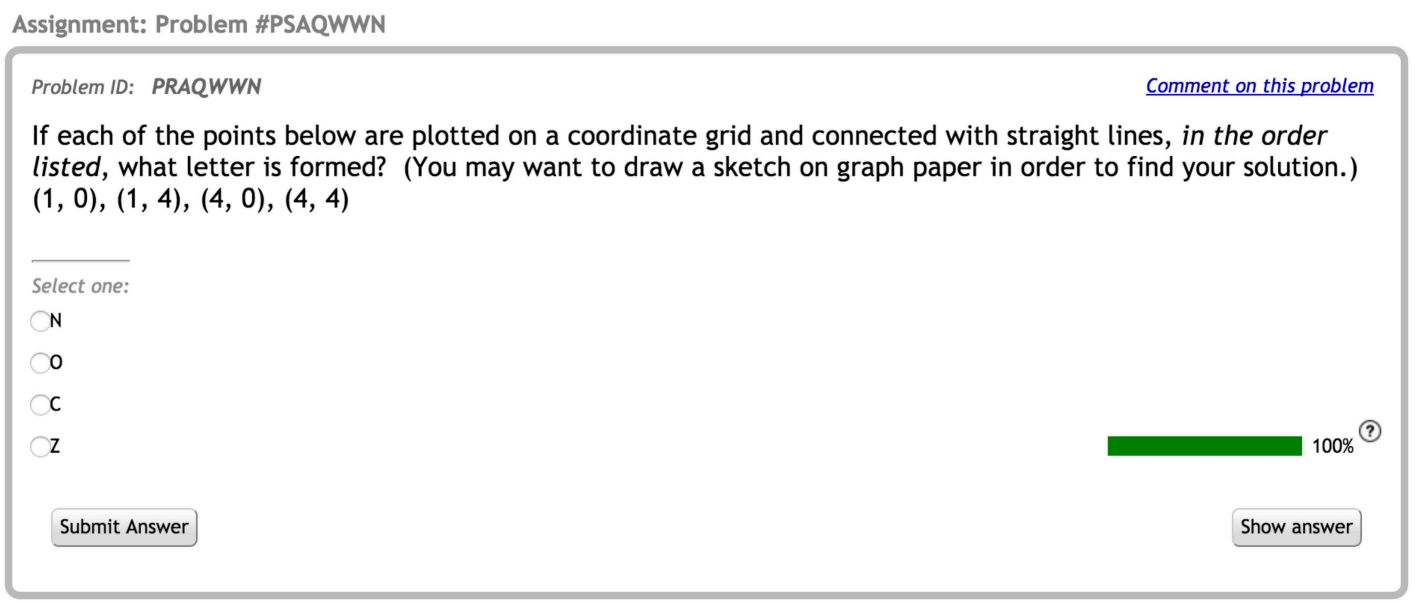

Related Patterns: The Representation Practice design pattern implements the Try It Yourself design pattern [Bergin et al. 2012], whose goal is to promote better understanding of a topic through exercises, but in the context of representing math-problems. Consider using the Image-Enhanced Problem design pattern to clearly express problems with confusing or ambiguous elements.

Students may still get stuck answering a problem if they make incorrect representations or if they are unable to make the correct inferences. The Represent It design pattern may help guide the student to make and interpret representations. Hints may help keep students motivated to answer the problem as described in the Build and Maintain Confidence design pattern [Bergin et al. 2012]. Create a diverse set of problems as described in the One Concept Several Implementations design pattern [Bergin et al. 2012] so that students are exposed to various problems that require different representations to solve. 


\section{Represent It}

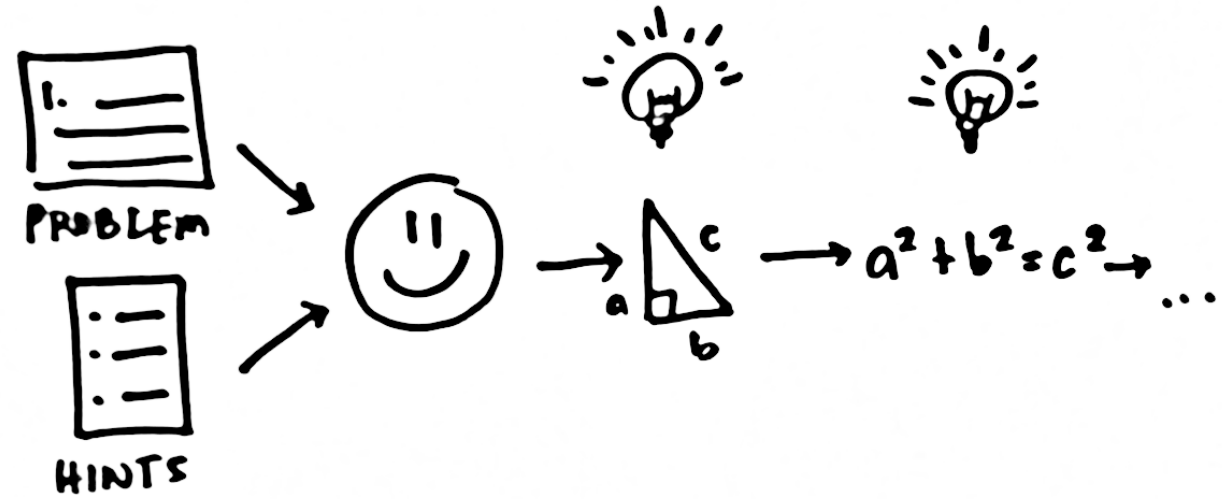

Context: Students are answering a math problem on an online learning system to help them understand a topic they recently learned.

Problem: Students find it difficult to solve problems when they think about solutions abstractly.

\section{Forces:}

- Increased difficulty. Solving problems mentally is difficult because working memory is limited. It becomes harder to process information as working memory becomes occupied by the different elements required to solve the problem.

- Careless mistakes. Students are susceptible to committing unintended errors when they try to solve problems mentally especially when they experience high cognitive load.

- Impaired metacognition. High cognitive load makes it difficult to manage the learning task. Students may have difficulty tracking their progress, evaluating their performance, or selecting effective learning strategies.

Solution: Therefore, encourage students to externalize their thoughts with representations that foster the understanding of the problem or leads to the correct answer. Online learning systems often provide facilities for automated feedback such as hints, error messages, or follow-up questions. These functionalities may be used to remind the student to write down notations, figures, tables, or other representations that may help visualize the problem and solution more concretely.

Provide just enough details about the representation for the student to use it. Too much information might make the problem too easy.

\section{Benefits:}

- Externalizing the elements of the problem frees up the working memory making it easier to process information.

- Compared to mental representations, it is easier to locate and address errors in externalized representations because they can be seen explicitly. Externalizing representations also frees up working memory, which facilitates focused processing.

- Students are better able to perform metacognitive tasks with less cognitive load. Metacognition helps students evaluate, assess, and revise their learning strategies. 


\section{Liabilities:}

- The online learning system needs to support feedback mechanisms to use the pattern (e.g., hint messages).

- The student should have some prior knowledge on using the representation otherwise they would not be able to use it.

- In some cases, there may be multiple representations that lead towards the problem's solution. The optimal order and necessary types of representations that are created and used may vary by student.

\section{Supporting Theories:}

- Writing down representations, just like drawing figures or notations, makes it easy to view problem details without keeping them all in memory [Polya 2014]. Using a more tangible medium may decrease cognitive load and make it easier to make inferences.

- It is difficult to complete a task when too much information is introduced because of working memory limitations [Sweller 2004].

- Expert guidance, such as providing hints, may help students achieve difficult tasks within the Zone of Proximal Development, which they are unable to do on their own [Hume et al. 1996, Vygotsky 1962].

- Desirable difficulty is beneficial to learning because activities that are too easy or do not challenge learners' understanding of a concept require less mental processing and often, less learning [Bjork 1994, Piaget 1952].

Known implementations: Math teachers often encourage students to think of a problem's representation and write it down to help them solve problems (e.g., "Draw a figure"). Teachers reinforce this skill by reminding students to use representations and make inferences from them (e.g., [McIntosh 2015, Noh et al. 2013, Polya 2014]). This is also seen in some online learning systems such as ASSISTments [Heffernan and Heffernan 2014], which provides hints that remind students to draw figures. Picture Algebra [Koedinger 2002] is another online learning system that encourages students to draw figures and asks questions regarding the figure they drew to help them solve a given problem.

The following figure shows an example of a hint in the ASSISTments online learning system. The hint reminds the student to draw a right triangle, which is the first step in the problem solving process. The hint provides enough information for students to recall the process and make inferences that may lead them to the solution. 


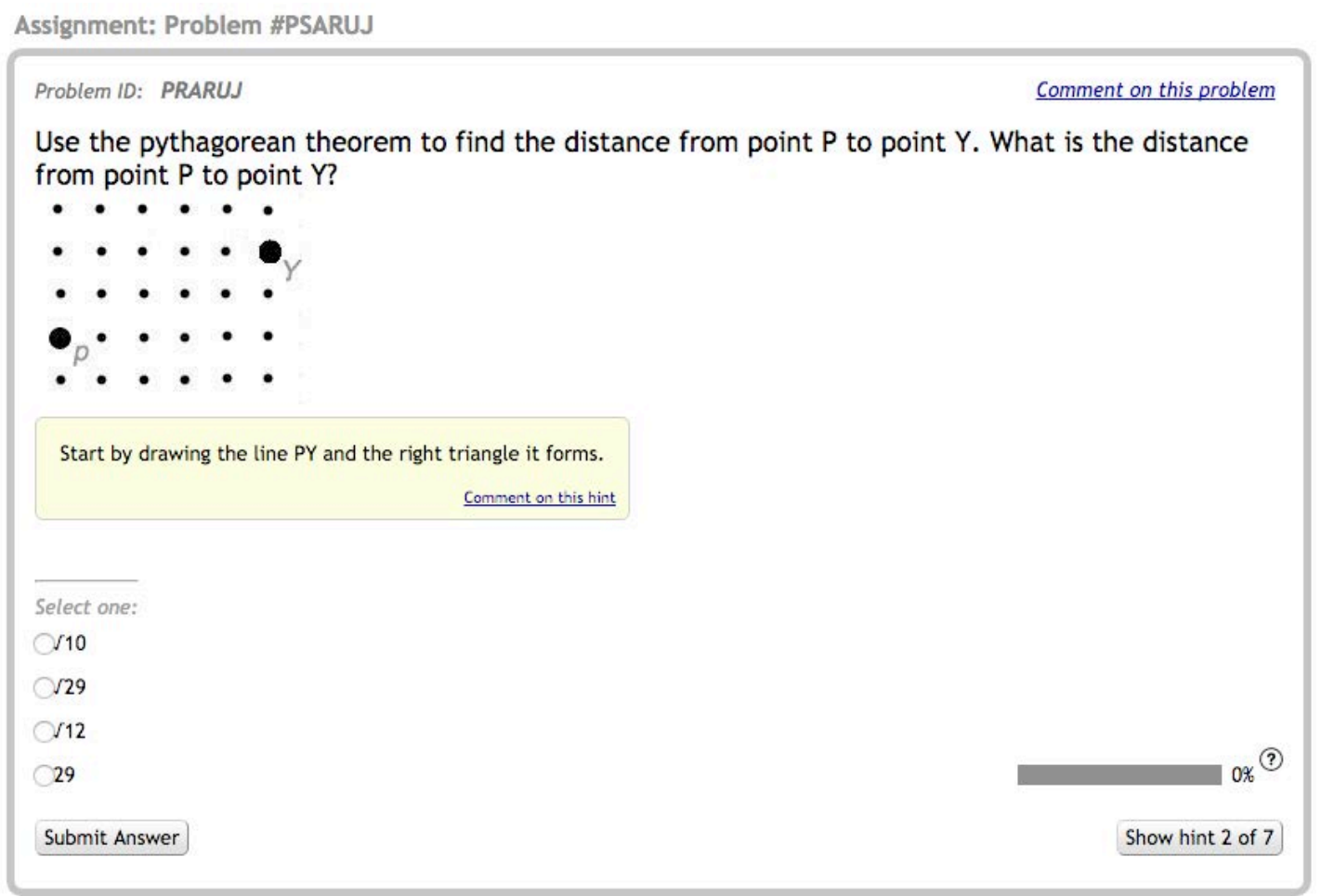

Related Patterns: The Represent It design pattern can be used while implementing the Representation Practice design pattern to prompt students to represent problems and their solutions. Images may be used to clarify hints if students are still unable to solve the problem as described in the Image-Enhanced Hint design pattern. Students may be allowed request for more hints when they need it, but only provide enough information each time to maximize their learning opportunity as described in the Increasing Hint Specificity design pattern. Figure 2 shows a map of the aforementioned patterns, which helps to explain how they are related. 


\section{Image-Enhanced Problem}

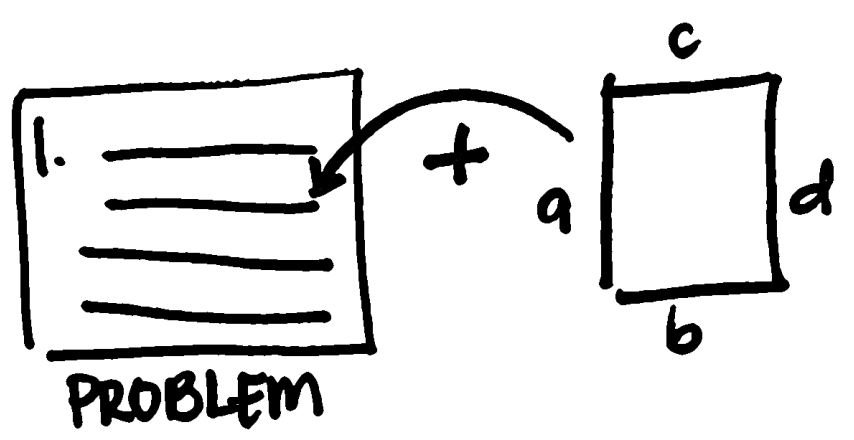

Context: A problem-solving activity is being designed for an online learning system to help students understand a topic they recently learned.

Problem: Students are unable to solve some problems that contain ambiguous terms and explanations.

\section{Forces:}

- Term familiarity. Some terms may have multiple meanings. Students may interpret a term the way they are most accustomed to, which can be different from the intended use. In some cases, students may not understand the term at all.

- High cognitive load. Students' cognitive load increase when they try to comprehend ambiguous terms and explanations aside from understanding and solving a problem.

- Time. Students may spend more time trying to understand the problem instead of solving it.

- Misunderstanding the problem. Students may end up solving for something unrelated if they misunderstand the problem.

- Unnecessary information. Providing elaborate explanations to clarify terms make the problem longer and can distract students from the actual problem.

Solution: Therefore, provide simple images and reference them in written text to clarify ambiguous terms and explanations. Do not add unnecessary details in images that may distract students from the focus of the problem or reveal learning objectives. Place the image close to the section of the written text that references it so that students do not need to scroll up and down the screen.

\section{Benefits:}

- Images can succinctly illustrate concepts that may clarify and disambiguate confusing terms and explanations.

- Students can focus their mental effort on solving the problem after they disambiguate terms and explanations.

- Clear and simple images can help students understand the problem quickly, which leaves them more time to solve it.

- Students are less likely to solve for something unrelated when they have a clear understanding of the problem.

- Well-designed images can express ideas concisely that will allow students to focus on the important elements of the problem. 


\section{Liabilities:}

- It takes more time and effort to create appropriate images for each problem.

- Some ideas, such as abstract concepts, are difficult to illustrate.

- Images take a longer time to load over the network compared to text.

- Images may not always appear on the screen as intended due to uncontrollable factors (e.g., screen size, color and screen resolution supported, image loading is disabled in the browser).

\section{Supporting Theories:}

- Long descriptive text can be expressed concisely with an image [Moyer 2010].

- Introducing unnecessary information and tasks increases cognitive load, which may make the task more difficult and less likely to be completed [Sweller 2004].

- The multimedia principle suggests the use of well-designed graphics and text to enhance understanding and learning [Clark and Mayer 2011, Fletcher and Tobias 2005].

Known implementations: Learning systems often use images to clarify written problems. Higher learning gains were observed when images and text were used to present problems compared to text alone on different domains such as Mathematics [Heffernan and Heffernan 2014, Moreno and Mayer 1999), Biology [Stull and Mayer 2007], and reading and comprehension [McCrudden et al. 2009].

The figure below shows a math problem for writing equations. The problem uses a golfanalogy and introduces the terms "tee" and "hole", which may be unfamiliar to some students. An image was used to illustrate the different problem elements, which may help students understand unfamiliar terms.

\section{Assignment: Problem \#PSA6AV7}
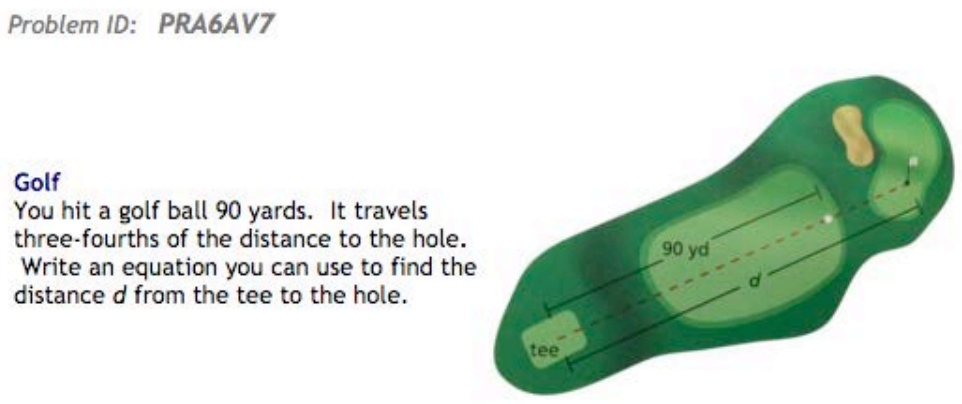

Type your answer below:

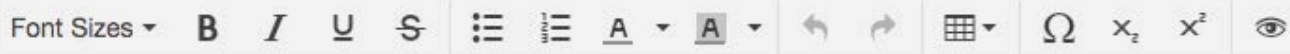


Related Patterns: The Image-Enhanced Problem design pattern can be used to clarify math problems that contain many details, which are easily confused. It can be used with the Representation Practice design pattern to provide students with essential images that can help clarify the problem without revealing too much information. Remember to use the Keep It Simple design pattern [Cunningham and Cunningham 2014] when designing images so that unnecessary details do not distract students.

Provide facilities such as those described in the Represent It and Image-Enhanced Hint design patterns to help students who still struggle to solve the problem. The use of images to facilitate understanding and hints to support student learning may help maintain student motivation as described in the Build and Maintain Confidence design pattern [Bergin et al. 2012]. 


\section{Image-Enhanced Hint}

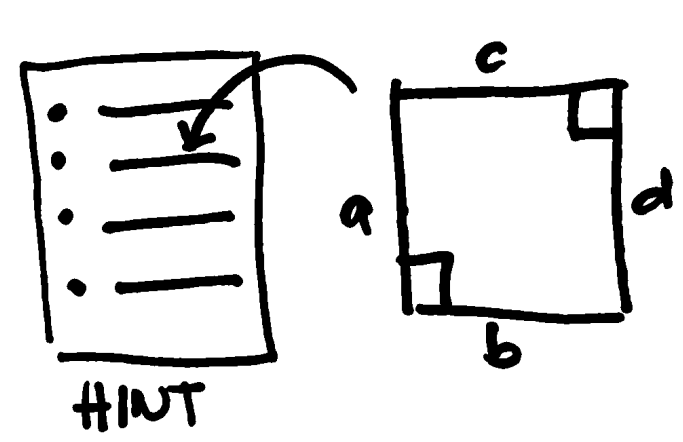

Context: A hint is being designed to provide feedback to students answering a problem-solving activity on an online learning system.

Problem: Students may be unable to understand hints that contain ambiguous terms and explanations.

\section{Forces:}

- Term familiarity. Some terms may have multiple meanings. Students may interpret a term the way they are most accustomed to, which can be different from the intended use. In some cases, students may not understand the term at all.

- High cognitive load. Hints may increase cognitive load when they include ambiguous terms and explanations that students need to understand before they can utilize the hint to solve the problem.

- Unnecessary information. Providing elaborate explanations to clarify terms make the hint longer and can distract students from the actual hint.

- Hint utility. If hints are too difficult to understand, students may move on to the next hint, disregard hints altogether, or stop solving the problem.

- Misunderstanding the hint. Hints may lead students to solve for something unrelated if they are unable to understand it correctly.

Solution: Therefore, provide clear and simple images and reference them in written text to clarify ambiguous terms and explanations in the hint. Do not add unnecessary details in images that may distract students from the focus of the hint or reveal learning objectives too quickly. For example, if part of the expected visualization (e.g., notation, equation, figure) needs to be shown in the image, reveal only the necessary parts, but leave the rest for the student to complete. Place the image close to the section of the written text that references it so that students do not need to scroll up and down the screen.

\section{Benefits:}

- Images can succinctly disambiguate confusing terms and explanations in the hints.

- Students can focus their mental effort on understanding the intention of the hint after they disambiguate terms and explanations.

- Well-designed images can express ideas concisely that will allow students to focus on the important elements of the hint.

- Clear hints are more likely to help students and motivate them to solve the problem.

- Students are less likely to solve for something unrelated when they have a clear understanding of the hint. 


\section{Liabilities:}

- It takes more time and effort to create appropriate images for hints.

- Some ideas, such as abstract concepts, are difficult to illustrate.

- Images take a longer time to load over the network compared to text.

- Images may not always appear as intended due to uncontrollable factors (e.g., screen size, color and screen resolution supported, image loading is disabled in the browser).

\section{Supporting Theories}

- According to the Zone of Proximal Development, expert guidance can help students achieve difficult tasks that they are not capable of completing on their own [Vygotsky 1962].

- Student learning can be scaffolded using different functions such as marking critical features (i.e., accentuate relevant features of the task) and demonstration [Wood et al. 1976].

- It is difficult to complete a task when too much information, such as long text explanations, is introduced because of working memory limitations [Sweller 2004].

- Student attention and patience is a limited resource possibly affected by pending deadlines, upcoming tests, achievement in previous learning experiences, motivation, personal interest, quality of instruction, and others [Arnold et al. 2005, Bloom 1974].

- According to the multimedia principle, learning can be enhanced by combining images and text [Clark and Mayer 2011, Fletcher and Tobias 2005].

- Desirable difficulty is beneficial to learning because activities that are too easy or do not challenge learners' understanding of a concept require less mental processing and often, less learning [Bjork 1994, Piaget 1952].

Known implementations: Math teachers often teach students to make representations that help them find the solution. When students have difficulty finding the solution, teachers may remind students to use visualizations or reveal parts of possible visualizations to help them (e.g., [McIntosh 2015, Noh et al. 2013, Polya 2014]). Online learning systems also employ a similar approach for supporting student learning. Cognitive Tutor Geometry provides students with practice problems and associated hints to support their learning [Aleven et al. 2006]. The hints provided by the system may be presented in written form or combined with images depending on the student's progress. Similarly, the ASSISTments online learning system allows students to request for hints that are presented in written form or combined with images depending on the intended design of the problem [Heffernan and Heffernan 2014]. Increasingly more information is presented as students request for more hints.

The following figure shows an example of a hint in the ASSISTments online learning system. The first hint encouraged the student to draw a figure. However, another hint was requested possibly because the student was still unable to make the appropriate visualization. The second hint shows the expected figure that the student should have drawn. The image is much easier to understand compared to an elaborate, possibly confusing, written description of the figure (e.g., Your figure should now have 3 lines: a diagonal line from point $\mathrm{P}$ to point $\mathrm{Y}$, a line from point $\mathrm{P}$ to the 5th point to the right of point $\mathrm{P}$, and a line from point $\mathrm{Y}$ to the second point below point $\mathrm{Y}$. The vertical line and horizontal lines should be perpendicular to each other to form a right triangle ...). 
Assignment: Problem \#PSARUJ

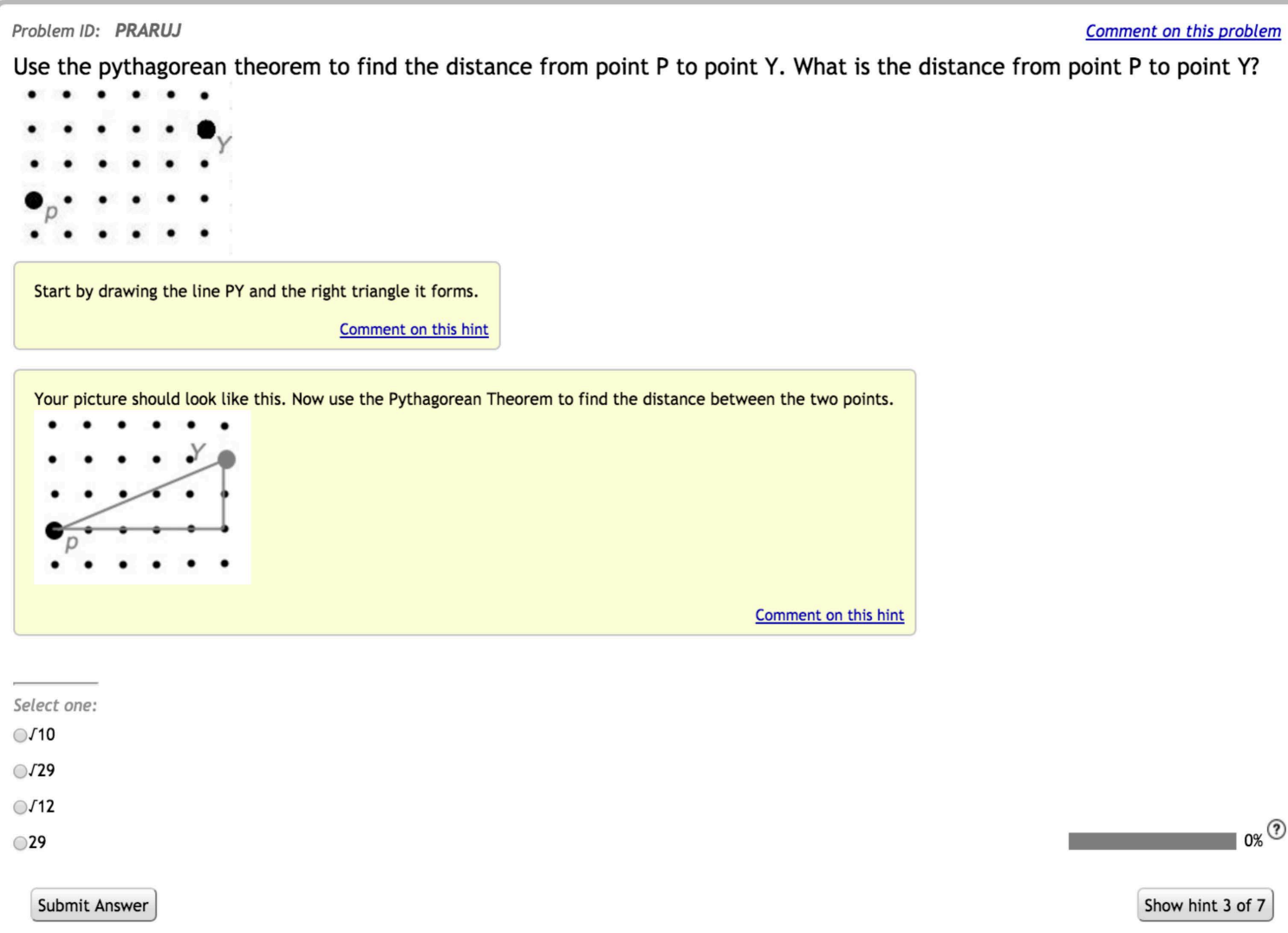

Related Patterns: The Image-Enhanced Hint design pattern can help clarify ambiguous terms and explanations in hints such as those created with the Represent It design pattern. Use the Keep It Simple design pattern [Cunningham and Cunningham 2014] when designing images so that unnecessary details do not distract students or reveal learning objectives too quickly. Using images to facilitate understanding may help maintain student motivation as described in the Build and Maintain Confidence design pattern [Bergin et al. 2012]. 


\section{SUMMARY AND NEXT STEPS}

The paper discussed four design patterns that focus on helping students visualize math problems in online learning systems: Representation Practice, Represent It, Image-Enhanced Problem, and Image-Enhanced Hint. Math teachers and content designers might find the patterns useful to guide the creation of high quality math learning activities in the context of an online learning system.

The patterns presented in this paper are part of a pattern language for math problems and corresponding learning support in online learning systems, which is currently under development. Some related patterns were presented in Inventado and Scupelli [2015a] and Inventado and Scupelli [2015b]. The design patterns are also compiled in an online design pattern repository (http://learningenvironmentslab.org/openpatternrepository) and work is being done to foster collaboration between design pattern authors, domain experts, and design pattern users to continue writing, evaluating, and refining design patterns.

\section{ACKNOWLEDGEMENT}

This material is based upon work supported by the National Science Foundation under DRL-1252297. We would like to thank our shepherd Allan Kelly for his invaluable advice and feedback on this work. We also thank Ryan Baker, Stefan Slater, and Jaclyn Ocumpaugh from Teachers College Columbia University, and Neil Heffernan, Eric VanInwegen, and Korinn Ostrow from Worcester Polytechnic Institute for helping us analyze the data and gain insights for developing the methodology. We thank members of the Learning Environments Lab: Alexandra Merski for her help exploring the existing math problems, design of revised math problems, and help setting up the randomized control trials; Rachael Chang for her help with the randomized control trials and visualization of the 3D2P process; and Sharris Francisco-Inventado for her help with the visualization of the Pattern Language for Math problems and Learning Support in Online Learning Systems.

\section{REFERENCES}

Aleven, V., Mclaren, B., Roll, I., and Koedinger, K. 2006. Toward meta-cognitive tutoring: A model of help seeking with a Cognitive Tutor. International Journal of Artificial Intelligence in Education, 16(2), 101-128.

Allen, E., and Seaman, J. 2015. Grade Level: Tracking Online Education in the United States. Babson Survey Research Group and Quahog Research Group, LLC.

Arnold, A., Scheines, R., Beck, J. E., and Jerome, B. 2005. Time and attention: Students, sessions, and tasks. In Proceedings of the AAAI 2005 Workshop Educational Data Mining (pp. 62-66).

Bergin, J., Eckstein, J., Völter, M., Sipos, M., Wallingford, E., Marquardt, K., Chandler, J., Sharp, H., and Manns, M.L. 2012. Pedagogical patterns: advice for educators. Joseph Bergin Software Tools.

Bjork, R.A. 1994. Memory and metamemory considerations in the training of human beings. In J. Metcalfe and A. Shimamura (Eds.), Metacognition: Knowing about knowing. (pp.185-205). Cambridge, MA: MIT Press.

Bloom, B. S. 1974. Time and learning. American psychologist, 29(9), 682.

Clark, R.C. and Mayer, R.E. 2011. E-learning and the science of instruction: Proven guidelines for consumers and designers of multimedia learning. John Wiley \& Sons.

Carlsson, D. 2004. A categorization of HCI patterns. Department of Computing Science, Umea University, Sweden.

Cunningham and Cunningham Inc. 2014. Keep it Simple. Retrieved from http://c2.com/cgi/wiki?KeepItSimple.

Dearden, A. and Finlay, J. 2006. Pattern languages in HCI: a critical review. Human Computer Interaction, 21(1), 49-102.

D'Mello, S. and Graesser, A. 2012. Dynamics of affective states during complex learning. Learning and Instruction, 22(2), 145157.

Fletcher, J. D., and Tobias, S. 2005. The multimedia principle. In R.E. Mayer (Ed.), The Cambridge handbook of multimedia learning (pp. 117-134). New York: Cambridge University Press.

Heffernan, N. T., and Heffernan, C. L. 2014. The ASSISTments Ecosystem: Building a platform that brings scientists and teachers together for minimally invasive research on human learning and teaching. International Journal of Artificial Intelligence in Education, 24(4), 470-497.

Hume, G., Michael, J., Rovick, A., and Evens, M. 1996. Hinting as a tactic in one-on-one tutoring. The Journal of the Learning Sciences, 5(1), 23-47. 
Inventado, P.S. and Scupelli, P. 2016a. Design Patterns for Helping Students to Learn to Visualize Math Problems in Online Learning Systems. In Proceedings of the 21st European Conference on Pattern Languages of Programs (EuroPLoP '16).

Inventado, P.S. and Scupelli, P. 2016b. Learning-Support Design Patterns for Student Interactions with Math Problems. In Proceedings of the 23rd Conference on Pattern Languages of Programs (PLoP'16).

Inventado, P.S. and Scupelli, P. 2015a. Towards an open, collaborative repository for online learning system design patterns. eLearning Papers, 42(Design Patterns for Open Online Teaching):14-27.

Inventado, P.S. and Scupelli, P. 2015b. Data-driven design pattern production: a case study on the ASSISTments online learning system. In Proceedings of the 20th European Conference on Pattern Languages of Programs (EuroPLoP '15). ACM, New York, NY, USA, Article 14, 13 pages.

Koedinger, K. R. 2002. Toward Evidence for Instructional Design Principles: Examples from Cognitive Tutor Math 6. In Proceedings of the Annual Meeting [of the] North American Chapter of the International Group for the Psychology of Mathematics Education, ERIC/CSMEE Publications, Columbus, OH, USA, pp. 21-49.

Koedinger, K. R., and Aleven, V. 2007. Exploring the assistance dilemma in experiments with cognitive tutors. Educational Psychology Review, 19(3), 239-264.

Koedinger, K. R., Kim, J., Jia, J. Z., McLaughlin, E. A., and Bier, N. L. 2015. Learning is Not a Spectator Sport: Doing is Better than Watching for Learning from a MOOC. In Proceedings of the Second (2015) ACM Conference on Learning@ Scale (pp. 111-120). ACM.

Köppe, C., Niels, R., Holwerda, R., Tijsma, L., van Diepen, N., van Turnhout, K., and Bakker, R. 2015. Flipped Classroom Patterns - Designing Valuable In-Class Meetings. Proceedings of the 20th European Conference on Pattern Languages of Programs, EuroPLoP'15. Irsee, Germany.

McCrudden, M. T., Schraw, G., and Lehman, S. 2009. The use of adjunct displays to facilitate comprehension of causal relationships in expository text. Instructional Science, 37(1), 65-86.

McIntosh, K.L. 2015. Montessori Mathematics Curriculum and Lower Elementary Students Understanding of Length Measurement. Masters of Arts in Education Action Research Papers. Paper 148.

Mitrovic, A. 2012. Fifteen years of constraint-based tutors: what we have achieved and where we are going. User Modeling and User-Adapted Interaction, 22(1-2), 39-72.

Mor, Y. 2010. Guess my X and other techno-pedagogical patterns: toward a language of patterns for teaching and learning mathematics. In: 13th European Conference on Pattern Languages of Programs(EuroPLoP 2008), 9-13 July 2008, Irsee, Germany, CEUR-WS, pp. 348-384.

Mor, Y., and Warburton, S. 2015. Practical Patterns for Active and Collaborative MOOCs: Checkpoints, FishBowl and See Do Share. eLearning, 48:48-56.

Moreno, R., and Mayer, R. E. 1999. Multimedia-supported metaphors for meaning making in mathematics. Cognition and instruction, 17(3), 215-248.

Moyer, D. 2010. Just Show It. The napkin sketch workbook. Blurb.

Noh, J., Warren, J., Huh, N., and Ko, H. K. 2013. Instruction Using Scaffolding for Language Learner Students in Solving Mathematical Word Problems. Research in Mathematical Education, 17(3), 169-181.

Piaget, J. 1952. The origins of intelligence. New York: International University Press.

Polya, G. 2014. How to Solve It: A New Aspect of Mathematical Method: A New Aspect of Mathematical Method. Princeton university press.

Renkl, A. 2014. The worked examples principle in multimedia learning. In R.E. Mayer (Ed.), The Cambridge handbook of multimedia learning (pp. 391-412). New York: Cambridge University Press.

Rohrer, D., and Taylor, K. 2006. The effects of overlearning and distributed practice on the retention of mathematics knowledge. Applied Cognitive Psychology, 20, 1209-1224.

Stamper, J., Barnes, T., and Croy, M. 2011. Enhancing the automatic generation of hints with expert seeding. International Journal of Artificial Intelligence in Education, 21(1-2), 153-167.

Stull, A. T., and Mayer, R. E. 2007. Learning by doing versus learning by viewing: Three experimental comparisons of learnergenerated versus author-provided graphic organizers. Journal of educational psychology, 99(4), 808.

Sweller, J. 2004. Instructional design consequences of an analogy between evolution by natural selection and human cognitive architecture. Instructional science, 32(1-2), 9-31.

Vygotsky, L. S. 1962. Language and thought. Massachusetts Institute of Technology Press, Ontario, Canada.

Warburton, S., and Mor, Y. 2015. A set of patterns for the structured design of MOOCs. Open Learning: The Journal of Open, Distance and e-Learning, 1-15.

Wood, D., Bruner, J. S., and Ross, G. 1976. The role of tutoring in problem solving. Journal of child psychology and psychiatry, 17(2), 89-100. 